\title{
Massive poles in Lee-Wick quantum field theory
}

\author{
John F. Donoghue \\ Department of Physics, University of Massachusetts Amherst, Massachusetts 01003, USA \\ Gabriel Menezes ${ }^{\dagger}$ \\ Department of Physics, University of Massachusetts Amherst, Massachusetts 01003, USA \\ and Departamento de Física, Universidade Federal Rural do Rio de Janeiro, \\ 23897-000 Seropédica, RJ, Brazil
}

(Received 14 January 2019; published 29 March 2019)

\begin{abstract}
Most discussions of propagators in Lee-Wick theories focus on the presence of two massive complex conjugate poles in the propagator. We show that there is, in fact, only one pole near the physical region or, in another representation, three polelike structures with compensating extra poles. The latter modified Lehmann representation is useful caculationally and conceptually only if one includes the resonance structure in the spectral integral. We treat both the photon propagator in Lee-Wick electrodynamics and the spin-two propagator in quadratic gravity.
\end{abstract}

DOI: 10.1103/PhysRevD.99.065017

\section{INTRODUCTION}

Lee and Wick have formulated a type of theory which is finite, yet yields all the usual predictions at low energy [1-3]. They endow new fields with a negative metric, with the result that the propagation of these fields cancels off the high-energy divergences of usual field theory. In rather simplistic terms, it is similar to including the Pauli-Villars regulators as the dynamical fields. For example the electromagnetic propagator is modified at tree level via

$$
\begin{aligned}
i D_{F \mu \nu}(q) & =-i g_{\mu \nu}\left[\frac{1}{q^{2}}-\frac{1}{q^{2}-\Lambda^{2}}\right] \\
& =-i g_{\mu \nu} \frac{-\Lambda^{2}}{q^{2}\left(q^{2}-\Lambda^{2}\right)}=-i g_{\mu \nu} \frac{1}{q^{2}\left(1-\frac{q^{2}}{\Lambda^{2}}\right)}
\end{aligned}
$$

The fact that the propagator goes asymptotically like $q^{-4}$ implies that loop integrals are not divergent. However, the massive field appears with negative norm-it is a ghost field. Once interactions are introduced, this dangerous feature is alleviated because the massive field decays into the light particles in the theory, such that it is not an asymptotic state in the spectrum. With some prescriptions for the treatment of loop integrals $[4,5]$, the theory appears

\footnotetext{
*donoghue@physics.umass.edu gabrielmenezes@ufrrj.br
}

Published by the American Physical Society under the terms of the Creative Commons Attribution 4.0 International license. Further distribution of this work must maintain attribution to the author(s) and the published article's title, journal citation, and DOI. Funded by SCOAP ${ }^{3}$. to be consistent and unitary, although there is a microscopic violation of causality on small scales [6-8]. This Lee-Wick mechanism for dealing with theories with quartic propagators is thought to be an important ingredient for many other higher derivative theories, including that of quadratic gravity [9-13]. Recent attempts to treat quadratic gravity as a fundamental quantum field theory [14-27] also have to address a negative norm state that appear in the graviton propagator and the analogy with Lee-Wick theories is quite close. It is, therefore, important to understand the underlying physics of Lee-Wick theories.

In these theories, when the massive state decays, the state develops a width. In most of the literature, the treatment involves a pair of poles that appear at the positions which are complex conjugates of each other, $q^{2}=M^{2}=m_{p}^{2}+i \gamma$ and $q^{2}=M^{* 2}=m_{p}^{2}-i \gamma$, with $m_{p}^{2}, \gamma$ both real. However, when explicit calculations are needed, one finds that there is only one pole. A representation with three poles-with two of them compensating -is also valid and useful. To our knowledge, the compensation of the latter poles was first noted in the context of an $O(N)$ model by Grinstein, O'Connell and Wise [8]. The purpose of this paper is to provide a clear discussion of this issue and to highlight the importance of the spectral integral in the latter representation.

\section{LOCATION OF THE POLE IN THE PROPAGATOR}

Both the usual photon and the heavy Lee-Wick particle couple to the electromagnetic current, and so the interaction can be described by a combined propagator. We start with a simple representation of this taken from the literature, which is quite intuitive and which captures the essence of 
the theory. We will later return to explain why this simple result is representative of the more complicated treatments that one finds when perusing the original literature. In Ref. [5], Boulware and Gross give the following representation for the propagator,

$$
i D_{F \mu \nu}\left(q^{2}\right)=-i g_{\mu \nu} D\left(q^{2}\right)
$$

with

$$
D\left(q^{2}\right)=\frac{1}{\left(q^{2}+i \epsilon\right)\left[1+\hat{\Pi}\left(q^{2}\right)-\frac{q^{2}}{\Lambda^{2}}\right]}
$$

with

$$
\begin{aligned}
\hat{\Pi}\left(q^{2}\right)= & q^{2} \frac{\alpha}{3 \pi} \int_{4 m_{f}^{2}}^{\infty} d s \frac{1}{s\left(s-q^{2}-i \epsilon\right)} \\
& \times \sqrt{1-\frac{4 m_{f}^{2}}{s}}\left(1+\frac{2 m_{f}^{2}}{s}\right) .
\end{aligned}
$$

Here, $\hat{\Pi}\left(q^{2}\right)$ is the finite part of the vacuum polarization function for a fermion of mass $m_{f}$, written in a dispersion theory representation. ${ }^{1}$ The propagator has been modified by integrating out the fermions, and the vacuum polarization yields a well-known correction factor. In transcribing this equation from Ref. [5], we have modified it to conform to our $(+,-,-,-)$ metric and also added the $i \epsilon$ in the denominator. This $i \epsilon$ is relevant in what follows because it helps determine the location of the pole. The location and sign of the $i \epsilon$ in the vacuum polarization is unambiguously determined by the usual placement of $i \epsilon$ in the fermion propagator.

The result is important in Lee-Wick theories because the vacuum polarization develops an imaginary part for large timelike $q^{2}$, which leads to a width for the massive ghostlike resonance. The fact that the massive ghost is unstable is crucial for the interpretation of the ghost, as it implies that the ghost does not appear in the asymptotic spectrum. Moreover, the other unusual properties of LeeWick ghosts, such as the opposite sign for the width, also follow from the form of the vacuum polarization.

\section{A. Numerical study}

First, let us display the content of the propagator using the vacuum polarization without any approximation and at a moderate coupling. Using a larger coupling allows one to visibly see the properties of the propagator which are harder to display if one uses a narrow width approximation. However, in this case, the mass and residue of the high mass pole must be found numerically. We will return to a useful analytic approximation at weaker coupling below.

\footnotetext{
${ }^{1}$ See, for example, problem V-3 in Ref. [28].
}

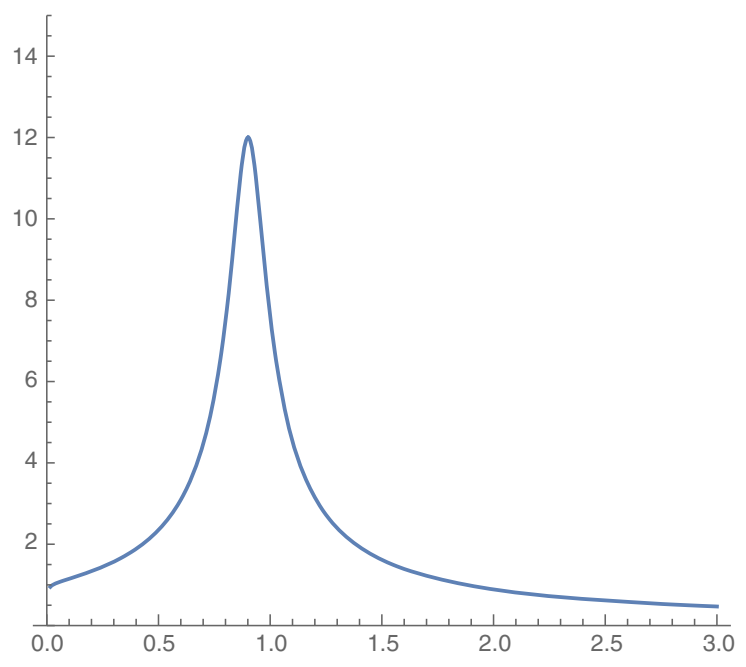

FIG. 1. The absolute value of the propagator as a function of $q^{2}$, using the parameters discussed in the text, in $\Lambda=1$ units, multiplied by $q^{2}$.

The vacuum polarization function for a fermion of mass $m_{f}$ has the form

$\hat{\Pi}\left(q^{2}\right)=-\frac{\alpha}{3 \pi}\left(1+\frac{2 m_{f}^{2}}{q^{2}}\right)\left[\sigma \log \left(\frac{1+\sigma}{1-\sigma}\right)-i \pi \sigma-\frac{5}{3}\right]$

for $q^{2}>4 m_{f}^{2}$, where

$$
\sigma=\sqrt{1-\frac{4 m_{f}^{2}}{q^{2}}}
$$

For smaller timelike values of $0 \leq q^{2} \leq 4 m_{f}^{2}$, it has the form

$$
\begin{aligned}
\hat{\Pi}\left(q^{2}\right)= & -\frac{2 \alpha}{3 \pi}\left(1+\frac{2 m_{f}^{2}}{q^{2}}\right)\left[\left(\sqrt{\frac{4 m_{f}^{2}}{q^{2}}-1}\right.\right. \\
& \left.\left.\times \operatorname{arccot}\left(\sqrt{\frac{4 m_{f}^{2}}{q^{2}}-1}\right)-1\right)+\frac{1}{6}\right] .
\end{aligned}
$$

Let us explore the propagator using $\alpha=0.25$, which is large enough to see the essential features. The value of $\Lambda$ can be chosen to set the overall scale of units, and our exploration sets $\Lambda=1$. For convenience, we choose the fermion mass to be $m_{f}^{2}=0.004$ in these units, small enough that threshold effects are not important near the pole but large enough that the logarithms of $q^{2} / m_{f}^{2}$ do not become so large as to affect the numerical accuracy.

The absolute value of the propagator is displayed in Fig. 1. Here, we have multiplied by a factor of $q^{2}$ so that a 

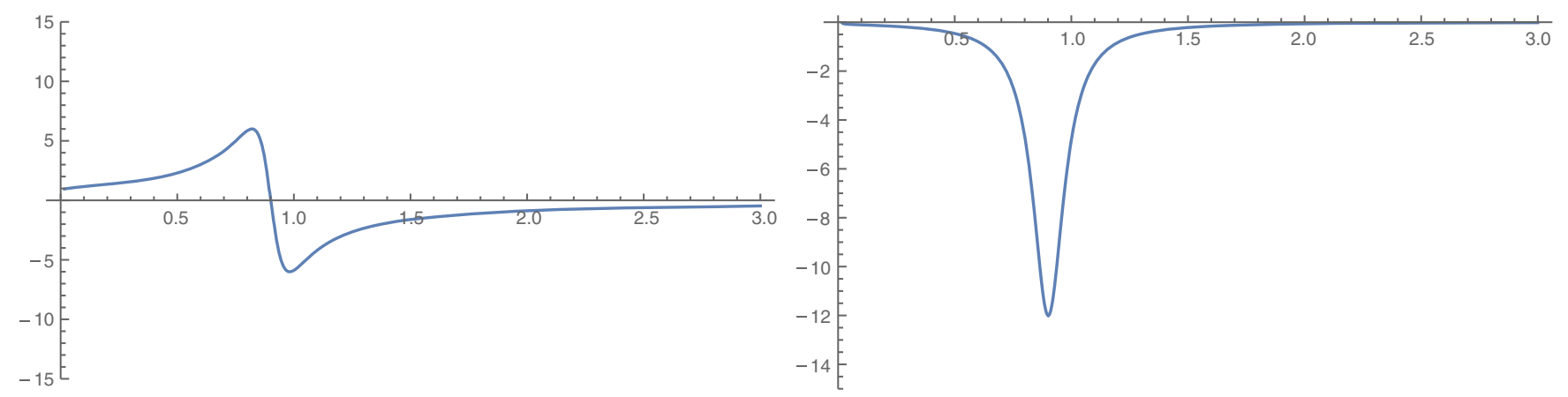

FIG. 2. Real and imaginary parts of the propagator, in the same format as Fig. 1.

normal photon propagator would be displayed as a flat line of magnitude unity. One can see the falloff at high values of $q^{2}$ as the Lee-Wick propagator asymptotically goes as $1 / q^{4}$. However, the most striking feature is obviously the resonance structure which is the unstable ghost resonance. The real and imaginary parts of the propagator are shown separately in Fig. 2. The zero in the real part and the peak in the imaginary part occur at $q^{2}=0.90116$ in $\Lambda=1$ units. The half-width at half-maximum of the imaginary part is 0.0809 in these units.

In the physical region, keeping $q^{2}$ real, we find that the resonance shape can be well approximated by the form

$$
D\left(q^{2}\right) \sim \frac{-\tilde{\beta}}{q^{2}-\mu^{2}-i \tilde{\gamma}}
$$

with the parameters

$$
\begin{aligned}
\mu^{2} & =0.90116 \\
\tilde{\gamma} & =0.0809 \\
\tilde{\beta} & =1.0772 .
\end{aligned}
$$

This propagator has two unusual signs in this region. The overall minus sign is the signal of the ghostlike nature. But also the imaginary part in the denominator is different from the usual structure for a propagator of an unstable particle. In the standard case, we normally have

$$
D(q)=\frac{1}{q^{2}-\left(M-i \frac{\Gamma}{2}\right)^{2}}=\frac{1}{q^{2}-\mu^{2}+i M \Gamma},
$$

where in this case $\mu^{2}=M^{2}-\Gamma^{2} / 4$, This sign difference in the width is a well-known feature of the Lee-Wick ghost. However, we note that because of the two sign differences, the imaginary part of the overall propagator is in agreement in the two cases,

$$
\operatorname{Im} D(q)=-\frac{\tilde{\beta} \tilde{\gamma}}{\left(q^{2}-\mu^{2}\right)^{2}+\tilde{\gamma}^{2}} v s-\frac{M \Gamma}{\left(q^{2}-\mu^{2}\right)^{2}+M^{2} \Gamma^{2}} .
$$

We reiterate that the sign of the width follows directly from the usual $i \epsilon$ prescription for the fermion propagator.

The parameters above were determined while keeping $q^{2}$ real. An alternate procedure would be to let $q^{2}$ become a complex variable, and to search for a pole in the propagator in the complex plane. If we follow this procedure and look in the neighborhood of the resonance of the previous paragraph, we find a pole described by the parameters

$$
D\left(q^{2}\right) \sim \frac{-\beta}{q^{2}-m_{p}^{2}-i \gamma},
$$

with

$$
\begin{aligned}
m_{p}^{2} & =0.901045 \\
\gamma & =0.08089 \\
\beta & =1.06922-0.09308 i
\end{aligned}
$$

The slight differences in the pole position and the residue between this determination and the previous one arises because the propagator is not purely quadratic in the momentum in this region, with the presence of the logarithm being important. We note that in the modified Lehmann representation, which we will discuss next, it is important to use this latter description of the pole parameters.

\section{B. Modified Lehmann representation}

It might be tempting to represent the propagator as the sum of two poles, for example,

$$
D(q) \sim \frac{1}{q^{2}}-\frac{1}{q^{2}-\mu^{2}-i \gamma} .
$$

This is accurate to a certain level, but has fundamental flaws. For example, it would give the propagator an imaginary part even at spacelike values of $q^{2}$, which violates general principles of QFT and also are not features of the true propagator. There is, however, an exact representation of the propagator in terms of poles and cuts which is very useful in elucidating the physical content of the theory. 


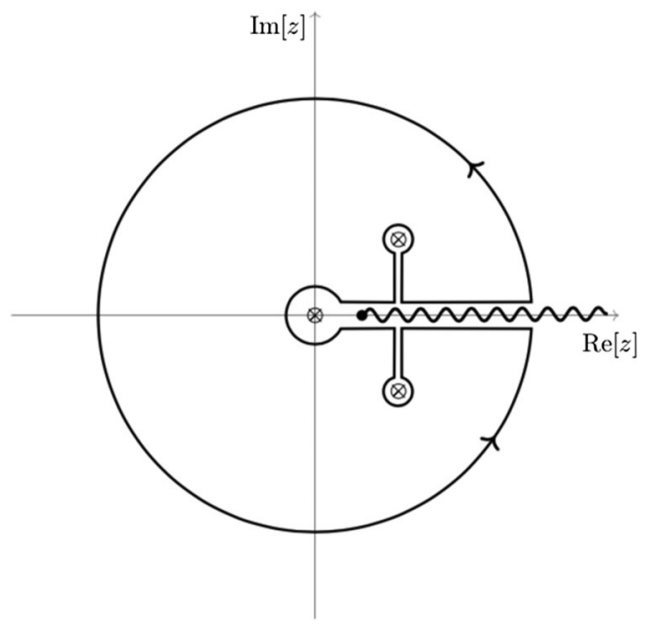

FIG. 3. The contour in the complex plane that yields the Lehmann representation. The poles are represented by encircled crosses, the branch cut is represented by a wavy line and the branch point is the black dot on the real axis. As discussed in the text, the cut begins at $q^{2}=4 m_{f}^{2}$.

In particular, it places the imaginary part of the propagator in a spectral integral, which is useful in the discussion of unitarity. To our knowledge, this modified Lehmann representation [29,30] was first introduced by Coleman in Ref. [6]. It has some unusual features which we aim to explain in this subsection, again using the numerical example given above.

If we consider the propagator as a more general function of complex $q^{2}$ we see four key features. There is, of course, the pole at $q^{2}=0$ and the cut running along the real axis from $q^{2}=4 m_{f}^{2}$ to $\infty$. We have discussed above the massive pole found slightly above the real axis. If we look on the other side of the cut, starting from $q^{2}-i \epsilon$ instead of $q^{2}+i \epsilon$, we find the complex conjugate location of the massive pole, i.e., at $q^{2}=m_{p}^{2}-i \gamma$. Because the propagator falls off fast enough at $\left|q^{2}\right| \rightarrow \infty$, we can use the Cauchy formula

$$
f\left(q^{2}\right)=\frac{1}{2 \pi i} \oint \frac{f(z)}{z-q^{2}} d z
$$

with the contour of Fig. 3 in order to write the identity

$$
\begin{aligned}
D(q)= & \frac{1}{q^{2}+i \epsilon}-\frac{\beta}{q^{2}-M^{2}}-\frac{\beta^{*}}{q^{2}-M^{* 2}} \\
& +\frac{1}{\pi} \int_{4 m_{f}^{2}}^{\infty} d s \frac{\rho(s)}{q^{2}-s+i \epsilon}
\end{aligned}
$$

where $\beta, \beta^{*}$ are the residues at the massive pole and the spectral function $\rho(s)$ is given by the discontinuity across the cut.

This representation satisfies the basic requirements of field theory. There is no imaginary part for spacelike momenta. The imaginary part coming from the massive

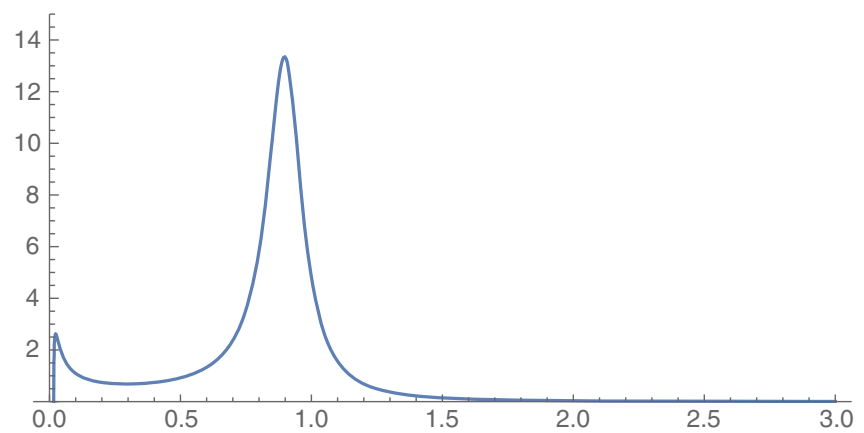

FIG. 4. The spectral function for $\alpha=0.25$ and $\Lambda=1$.

pole is cancelled by the complex conjugate pole. The only imaginary parts arise from the spectral function integral, which has an imaginary component only for $q^{2}>4 m_{f}^{2}$. The existence of two complex-conjugated poles is a well-known property of Lee-Wick theories. The spectral function contribution is, however, equally important and is much less discussed in the literature. We will see that it is also polelike in an important way.

We can isolate the spectral function by taking the imaginary part of the propagator

$$
-\left.\operatorname{Im} D(q)\right|_{q^{2}=s}=\rho(s) .
$$

This produces

$$
\rho(s)=\frac{\operatorname{Im} \hat{\Pi}(s)}{s\left[\left(1-\frac{s}{\Lambda^{2}}+\operatorname{Re} \hat{\Pi}(s)\right)^{2}+(\operatorname{Im} \hat{\Pi}(s))^{2}\right]}
$$

In the region near the massive pole, the imaginary part of the vacuum polarization is approximately a constant

$$
\operatorname{Im} \hat{\Pi}(s) \sim-\frac{\alpha}{3} \text { for } q^{2} \gg 4 m_{f}^{2} .
$$

This gives the spectral function a shape which is very close to a Breit-Wigner form. The result is shown in Fig. 4 for the parameters of the previous subsection. We have verified numerically that the use of this spectral function along with the pole results given by the determination of Eq. (13) reproduces the real and imaginary parts of the propagator within the numerical accuracy of the calculation.

These results appear to raise a puzzle. Our exploration of the original propagator revealed a single pole which governed the behavior in the physical region. The modified Lehmann representation involves three massive polelike structures, i.e., the pole and the complex conjugate pole as well as the spectral function. How can these describe the same physical content? The answer is that two of these ingredients almost cancel each other. We now demonstrate this feature.

The three massive components of the propagator can be studied separately. The real parts of each are shown in 


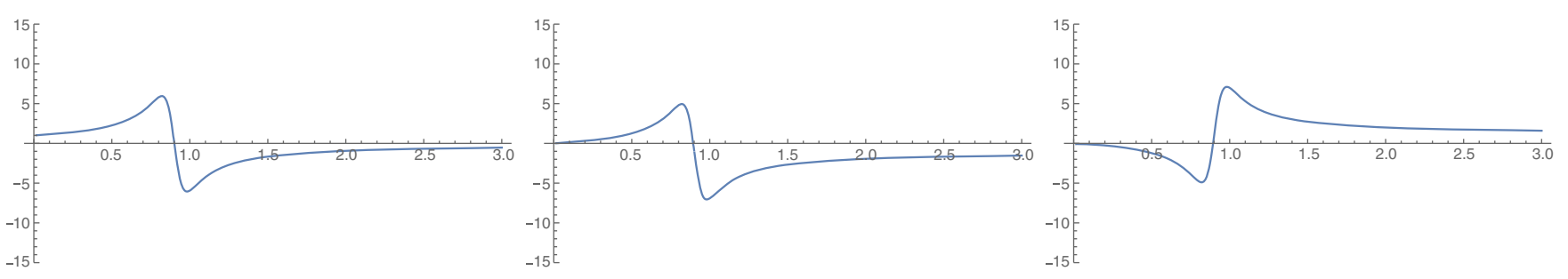

FIG. 5. Real parts of the three components of the propagator (left to right), the pole, the complex conjugate pole and the spectral contribution.
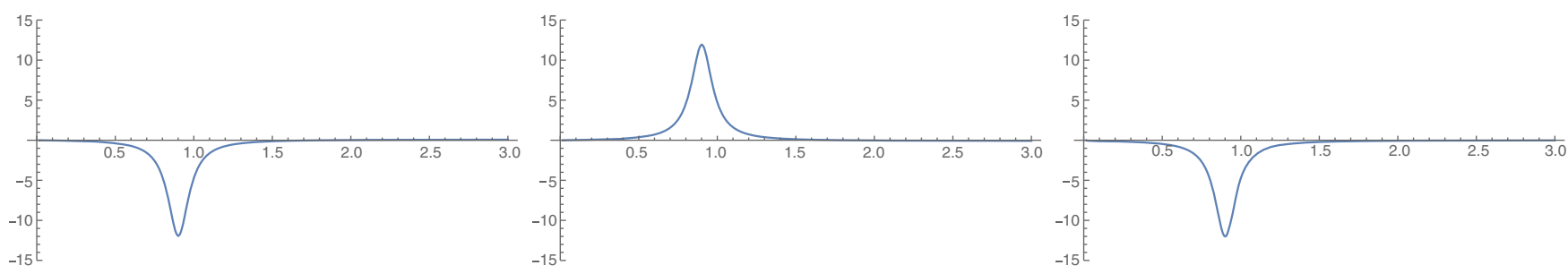

FIG. 6. Imaginary parts of the three components of the propagator (left to right), the pole, the complex conjugate pole and the spectral contribution.

Fig. 5 and the imaginary parts in Fig. 6 for our benchmark parameters. The easy comparison is that the pole and the complex-conjugate pole have the same real parts and opposite imaginary parts, as clearly required. The more interesting comparison is that the complex-conjugate pole and the spectral contribution appear to the eye to be opposite for both the real and imaginary parts. In practice, they are not exactly equal. The sum of these two components is displayed in Fig. 7. Note the change in scale on the vertical axis. There is a small residual which is required to properly describe the momentum dependence of the propagator away from the poles. But the important factor is that there is no polelike feature in the difference. The pole structure of the complex-conjugate pole is fully removed by the contribution of the spectral function.

This important role of the spectral function is missing from the early Lee-Wick literature. As far as we know, this cancelation was first noted in Ref. [8].

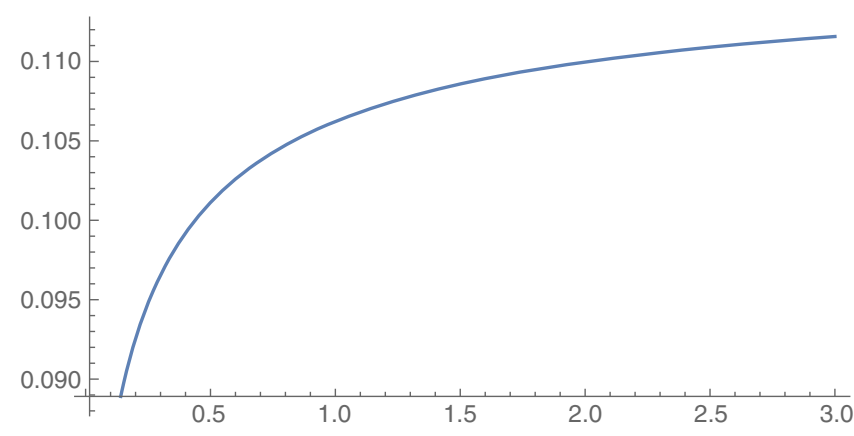

FIG. 7. The absolute value of the sum of the complex-conjugate pole contribution and the spectral function contribution to the propagator, multiplied by $q^{2}$, using the parameters of the preceding figures. The effect of the resonance cancels in the sum, leaving a small residual.
Note also that the spectral integral is important in the sum rule that determines the asymptotic behavior of the propagator. Since in Lee-Wick theories the propagator falls off as $1 / q^{4}$ at high momentum, and each of the components in the modified Lehmann representation falls off as $1 / q^{2}$, one must have the normalization sum rule [6]

$$
0=1-\beta-\beta^{*}+\frac{1}{\pi} \int_{4 m_{f}^{2}}^{\infty} \rho(s) d s
$$

Since $\beta, \beta^{*}$ are both near unity, the spectral integral can never be neglected. For our parameters, we find

$$
\frac{1}{\pi} \int_{4 m_{f}^{2}}^{\infty} \rho(s) d s=1.13844
$$

so that the normalization sum-rule is numerically satisfied.

Despite the near cancelation of two of the main ingredients of the modified Lehmann representation, the representation is important conceptually. This is largely because it demonstrates that the imaginary parts of the propagator are "normal". When dealing with the unstable ghost, we found that the imaginary part of the pole position was opposite of the usual expectation. Both the ghostlike nature and the imaginary part of the pole position lead to concerns about unitarity and the stability of the theory. However, in the modified Lehmann representation we see that the imaginary parts behave normally. They follow from a positive definite spectral function, with the usual $i \epsilon$ prescription in the denominator. The imaginary parts that come from the two ghostlike poles cancels each other. While there remain novel aspects of Lee-Wick theories, this feature is responsible for the indication that unitarity is still preserved in such theories. 


\section{Analytic approximation for weak coupling}

In Lee-Wick theories, the most important role of the vacuum polarization is to provide a width for the high mass ghost state, such that it is unstable. This feature arises at first order in $\alpha$. However, if one tries to solve exactly for the pole position and residue, one finds that they are nonlinear in $\alpha$. Moreover, there is a large logarithm of the form $\alpha \log \left(\Lambda^{2} / m_{f}^{2}\right)$. For small coupling, it would be preferable to treat the propagator to the leading relevant order in the coupling, and treat further corrections in perturbation theory. Such a treatment has a few modest subtle features, such as the fact that the normalization sum-rule of Eq. (20) will only be satisfied to order $\alpha$. We describe the perturbative treatment in this section.

The origin of the large logarithm comes from the choice to define the renormalized electric charge at $q^{2}=0$. The residual vacuum polarization function has been defined such that $\hat{\Pi}\left(q^{2}=0\right)=0$. At large values of $q^{2}$ it has the asymptotic expansion

$$
\hat{\Pi}\left(q^{2}\right)=-\frac{\alpha}{3 \pi}\left[\log \left(-q^{2} / m_{f}^{2}\right)-\frac{5}{3}\right]
$$

Physically this corresponds to the effect of a running coupling constant. To deal with it one can include the coupling constant in the propagator. More explicitly, at large positive $q^{2}$

$$
\alpha D\left(q^{2}\right) \sim \frac{\alpha}{q^{2}\left[1-\frac{\alpha}{3 \pi}\left[\log \left(q^{2} / m_{f}^{2}\right)-\frac{5}{3}\right]-\frac{q^{2}}{\Lambda^{2}}+i \frac{\alpha}{3}\right]}
$$

Written in terms of the running coupling defined at the value $q^{2}=\Lambda^{2}$, we have instead

$$
\alpha D\left(q^{2}\right) \sim \frac{\alpha(\Lambda)}{q^{2}\left[1-\frac{\alpha}{3 \pi} \log \left(q^{2} / \Lambda^{2}\right)-\frac{q^{2}}{\Lambda^{2}}+i \frac{\alpha}{3}\right]}
$$

to first order in the coupling. The large logarithm has been absorbed into the coupling and the remaining logarithmic running is weak near the pole.

Locating the pole is now easy to first order in the coupling. The real part of the propagator vanishes at $q^{2}=\Lambda^{2}$ for real $q^{2}$. It remains at this value to first order in $\alpha$ even when $q^{2}$ is allowed to be complex. The resonance parameters at leading order are

$$
\begin{aligned}
M^{2} & =m_{p}^{2}+i \gamma \\
m_{p}^{2} & =\Lambda^{2} \\
\gamma & =\frac{\alpha}{3} \\
\beta & =1 .
\end{aligned}
$$

At weak coupling, the poles and the spectral function are very narrow. For the physical value of $\alpha$, but other parameters unchanged from the previous section, the spectral function

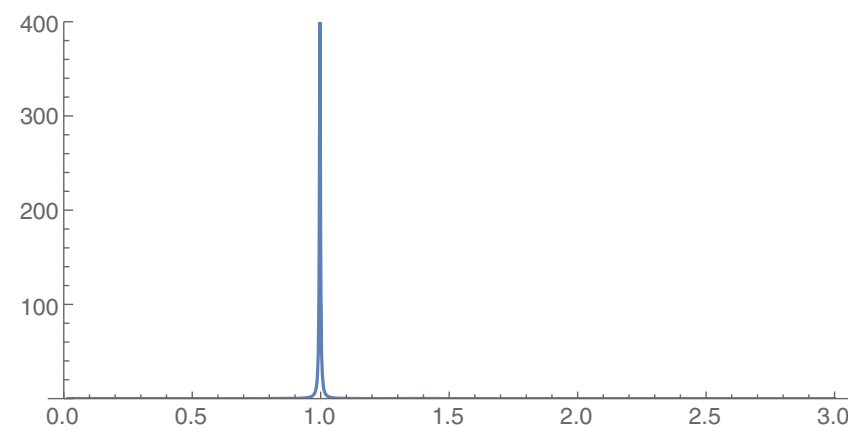

FIG. 8. The spectral function for $\alpha=1 / 137$.

is shown in Fig. 8. Nevertheless, when it is integrated over all values of $s$, the result differs from unity by an amount of order $\alpha$. In this case, the normalization sum rule is not satisfied exactly. However, given that the spectral function is so highly peaked and narrow, one can easily use a normalized Breit-Wigner distribution as an approximation, which then satisfies the sum rule.

The modified Lehmann representation continues to have three massive polelike structures: the physical pole, its complex conjugate and the Breit-Wigner of the spectral function integral. In the narrow width limit, the spectral integral can be done explicitly. Not surprisingly, it describes a massive pole

$$
\frac{1}{\pi} \int_{4 m_{f}^{2}}^{\infty} d s \frac{\rho(s)}{q^{2}-s+i \epsilon} \sim \frac{1}{q^{2}-m_{p}^{2}+i \gamma}
$$

and the cancellation with the complex conjugate pole becomes exact.

While we have demonstrated this cancellation at a particular value of the coupling constant at strong coupling, and at weak coupling, we emphasize that this it true for all values of the coupling constant.

\section{EXTENDING THE LEE-WICK SECTORS}

Our starting point, Eq. (3) from [5], captures the key feature of the Lee-Wick theory. However, in general, such theories are more complicated. For example, Lee-Wick QED also contains a heavy fermion ghost which appears in the fermion propagator [3]. If that ghost were light enough, it could modify the imaginary part of the vacuum polarization function at energies below the photon ghost pole. So our starting point corresponds to the case where the $\Lambda$ parameter for the heavy fermion ghost is larger than that in the photon propagator. Loops of the heavy fermion would renormalize the parameters of the low energy limit but would not generate an imaginary piece in the propagator at these energies, and our analysis of the pole structure would be unchanged.

To leading order, the analysis of poles in the fermion propagator would share the same features as the photon analysis above. The heavy fermion ghost picks up an imaginary part due to the coupling to the light states in the 
theory. There would be a modified Lehmann representation for the fermion propagator also, with a similar result.

If the heavy fermions were lower in mass than the photon ghost, unitarity requires that they generate imaginary parts also in the vacuum polarization. This would modify the location of the pole and the width, but would leave the rest of the analysis unchanged. It is expected that Lee-Wick theories are unitary. The clearest calculation that we know of demonstrating unitarity and showing the role of imaginary parts from heavy ghosts is in Ref. [8] within $O(N)$ theories.

While the focus of our work is on the structure of the theory, we note that if one takes the Lee-Wick QED model seriously, the massive states must be heavier than energies probed by the LHC, which presently stands at about $M \sim 4 \mathrm{TeV}$. The heavy photon resonance would be a narrow state with width $\Gamma \sim \alpha M / 3$. The associated microcausality violation $[3,6,8]$ would then be associated with a time scale of $\tau \sim 1 / \Gamma \sim 10^{-25}$ seconds, which is far shorter than any measurable time difference at present. ${ }^{2}$ In the gravitational case, which we will turn to next, the microcausality violation would be proportional to the Planck time, $10^{-43}$ seconds, which is much shorter still.

\section{QUADRATIC GRAVITY}

As mentioned in the introduction, much of the present interest in Lee-Wick theories occurs because gravitational quantum field theories have a similar structure when treated as fundamental theories in the ultraviolet region. Loops of matter fields coupled to the metric lead to divergences proportional to terms in the action which are quadratic in the curvatures [32]. There needs to be terms in the action with this structure in order to renormalize the divergences. Unlike the Lee-Wick formulation of QED, quadratic gravity is not finite, but it is renormalizeable [9]. One then naturally ends up with an acton containing the Einstein action linear in the curvature as well as quadratic curvature terms. Because the curvatures involve second derivatives of the metric, the propagators will involve both $q^{2}$ and $q^{4}$ terms. The gravitational vacuum polarization will add an imaginary component to the propagator for timelike $q^{2}$. This leads us to a Lee-Wick structure. In this section, we show how the spin-two propagator has the same structure as Lee-Wick QED theory described above.

In four dimensions, discarding a total derivative term, the general action to quadratic order is

$S_{\text {quad }}=\int d^{4} x \sqrt{-g}\left[\frac{2}{\kappa^{2}} R+\frac{1}{6 f_{0}^{2}} R^{2}-\frac{1}{\xi^{2}}\left(R_{\mu \nu} R^{\mu \nu}-\frac{1}{3} R^{2}\right)\right]$.

Here $\kappa^{2}=32 \pi G$. We have dropped the cosmological constant and will be expanding about Minkowski spacetime, $g_{\mu \nu}=\eta_{\mu \nu}+\kappa h_{\mu \nu}$.

\footnotetext{
${ }^{2}$ More specific phenomenology is addressed in Ref. [31].
}

The most interesting and dangerous component of the propagator is the spin-two portion, ${ }^{3}$ which contains the usual graviton at $q^{2}=0$ and a ghostlike resonance at higher timelike $q^{2}$. When the gravitational vacuum polarization is included in the propagator, it has the form

$$
\begin{aligned}
i D_{\mu \nu \alpha \beta} & =i \mathcal{P}_{\mu \nu \alpha \beta}^{(2)} D_{2}(q) \\
D_{2}^{-1}(q) & =q^{2}+i \epsilon-\frac{\kappa^{2} q^{4}}{2 \xi^{2}(\mu)}-\frac{\kappa^{2} q^{4} N_{\text {eff }}}{640 \pi^{2}} \ln \left(\frac{-q^{2}-i \epsilon}{\mu^{2}}\right)
\end{aligned}
$$

Here, $\mathcal{P}_{\mu \nu \alpha \beta}^{(2)}$ is the spin-two projector [17]. The coefficient of the logarithm, $N_{\text {eff }}$, is a number that depends on the number of light degrees of freedom with the usual couplings to gravity, ${ }^{4} N_{\text {eff }}=N_{V}+\frac{1}{4} N_{F}+\frac{1}{6} N_{S}+21 / 6$, where $V, F, S$ refer to gauge bosons, fermions and scalars, and the final number is due to low energy gravitons. With the Standard Model fields plus gravity, $N_{\text {eff }}=325 / 12$, and we use this number in the figures which we display. The coupling $\xi$ has been renormalized and has become a running coupling $\xi(\mu)$ where $\mu$ is the scale factor introduced in dimensional regularization, such that the propagator is independent of $\mu$. The main substantive difference of this propagator and that of the QED example given above is that the gravitational vacuum polarization is proportional to $q^{4}$, and hence is even better behaved at low energy than the QED example.

Because of the $i \epsilon$ in the logarithm, the propagator picks up an imaginary part. Much like the QED model discussed above, there is a massive ghostlike resonance in this propagator. At weak coupling it is located at

$$
M^{2}=m_{p}^{2}+i \gamma, \quad m_{p}^{2}=\frac{2 \xi^{2}\left(m_{p}\right)}{\kappa^{2}}, \quad \gamma=\frac{2 \xi^{2} m_{p}^{2} N_{\mathrm{eff}}}{640 \pi}
$$

At weak coupling, the residue at this location is -1 .

This propagator also has a modified Lehmann representation of the form of Eq. (16). By matching the imaginary part of the propagator to that of the Lehmann representation, one finds the spectral function,

$$
\rho(s)=\frac{\frac{N_{\text {eff }} K^{2}}{640 \pi}}{\left(1-\frac{s}{m_{r}^{2}}-\frac{N_{\text {eff }} K^{2} s}{640 \pi^{2}} \log \frac{s}{m_{r}^{2}}\right)^{2}+\left(\frac{N_{\text {eff }} K^{2} s}{640 \pi}\right)^{2}}
$$

This is close to that of a Breit-Wigner shape. For example, for $\xi^{2}=0.1$, the spectral function describes a very narrow resonance, shown in Fig. 9. As demonstrated in Sec. II C, at weak coupling, the resonance effect from the spectral

\footnotetext{
${ }^{3}$ For more on the decomposition of the propagator, see [17,33].

${ }^{4}$ We have simplified the result slightly in regards the contribution of graviton loops, but this has no effect on our discussion. A more complete treatment is found in [17].
} 


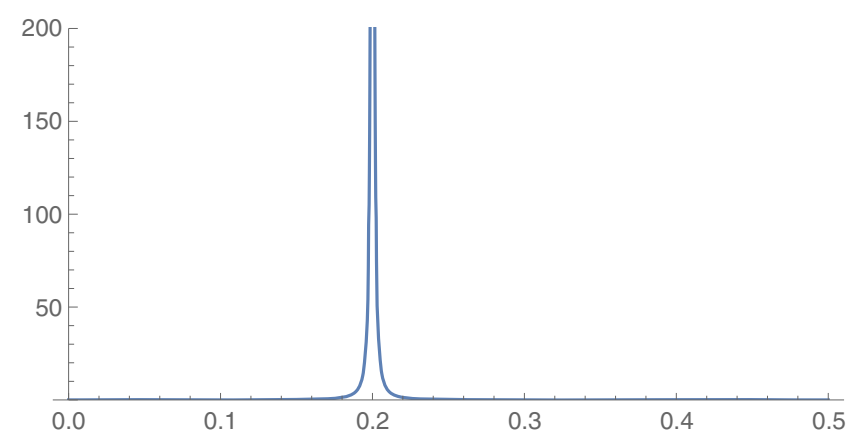

FIG. 9. The spectral function for $\xi^{2}=0.1$.

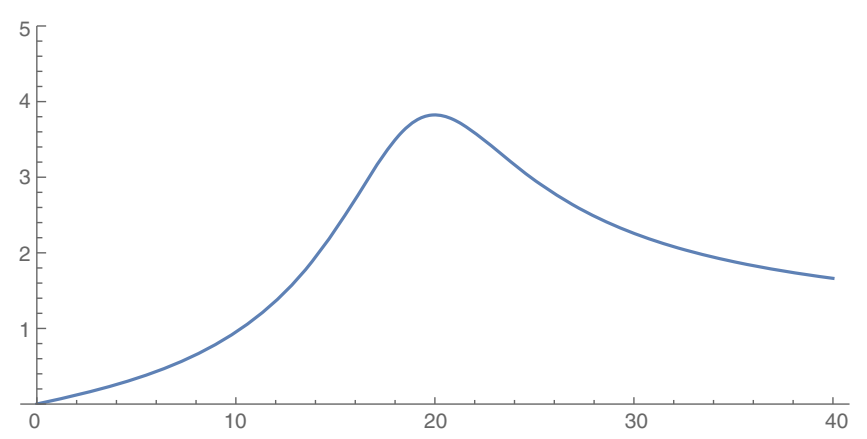

FIG. 10. The spectral function for $\xi^{2}=10$.

function cancels the effect of the complex conjugate pole. The calculation proceeds identically to that of Sec. II C.

In general, the compensation of the complex-conjugate pole and the spectral function is not exact. In order to better explain the differences, it is again useful to go to strong coupling where one can illustrate the features graphically. For example, we will present results for $\xi^{2}=10$. At large coupling, we solve for the parameters of the pole numerically. For $\xi^{2}=10$, in units where $\kappa=1$

$$
\begin{aligned}
M^{2} & =18.9+4.7 i \\
\beta & =0.95+0.17 i
\end{aligned}
$$

The spectral function retains the same functional form, but is not as sharply peaked. It is shown in Fig. 10. The corresponding propagator is plotted in Fig. 11.

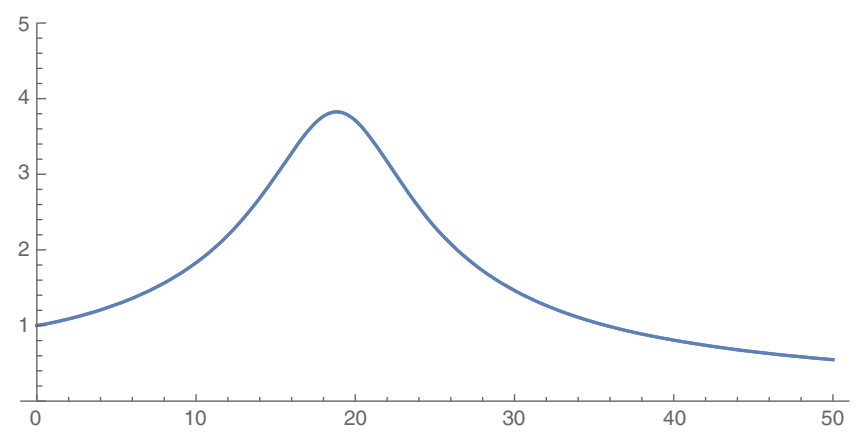

FIG. 11. The absolute value of the propagator, multiplied by $q^{2}$, for $\xi^{2}=10$.

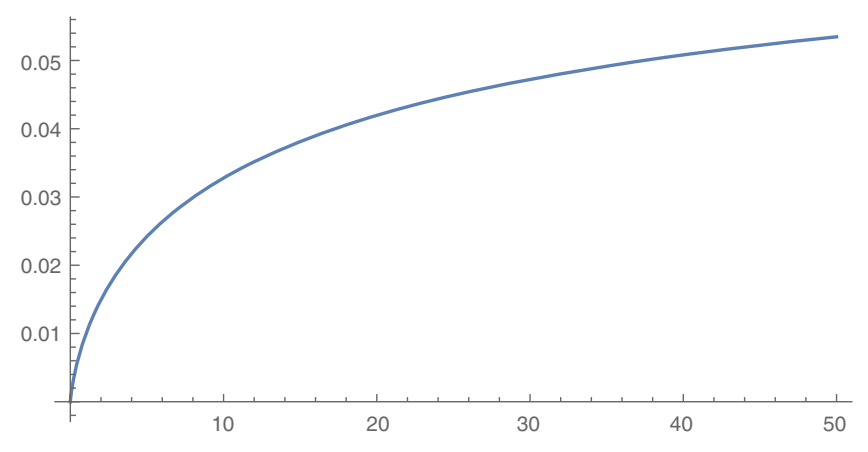

FIG. 12. The absolute value of the sum of the complexconjugate pole contribution and the spectral function contribution to the propagator, multiplied by $q^{2}$, for $\xi^{2}=10$. There is no resonant behavior in the sum of these contributions.

Again the cancelation of the sum of the complexconjugate pole and the spectral function is almost complete. We do not need to display the real and imaginary parts of each component, but they are visually similar to those of the QED example. Most importantly, if we add together the complex conjugate pole and the effect of the spectral integral we find the residual plotted in Fig. 12. While the cancelation between them is not complete at this strong coupling value, we note in particular that there is no resonant behavior in this sum.

Overall, we have shown that the quadratic gravity shares the same essential features in its spin-two propagator as is seen in the photon propagator for Lee-Wick QED.

\section{SUMMARY}

Most discussions of Lee-Wick theories emphasize the existence of an unstable massive ghost pole and its complex conjugate. The point of this paper is to emphasize that the spectral function also has a polelike structure-it is close to a Breit Wigner shape. In addition, the effect of the spectral integral is to cancel the resonance behavior of the complex-conjugate pole, leaving in general a small (nonresonant) residual.

Nevertheless, the modified Lehmann representation is conceptually and calculationally useful. The imaginary parts of the propagator at high energy lie in the spectral integral, which has the usual $i \epsilon$ structure. It is important for real calculations to include the spectral integral when discussing loops. We do not address here anything about the unusual contours chosen when evaluating Feynman integrals in these theories. However, the Lehmann representation captures the physics of Lee-Wick theories when one includes the resonance structure in the spectral function.

Much of the present interest in Lee-Wick theories comes from the study of quantum gravity [14-27]. If it is possible to describe a UV complete theory of quantum gravity using renormalizable quantum field theory, the gravitational interaction will necessarily involve higher derivatives in the fundamental action. This in general leads to high mass 
ghostlike states. Analogous to the discussion above, these states will be unstable and will decay into the light particles of the theory. The propagators of the gravity theory will be similar to that described above, with some differences due to the momentum dependence in the gravitational vacuum polarization. In particular, the compensation found in the modified Lehmann representation and the need for a resonance structure in the spectral integral will be the same. The understanding of the propagator is an important first step in the exciting possibility that quantum gravity can be described by a renormalizable quantum field theory.

\section{ACKNOWLEDGMENTS}

We are grateful for correspondence on these topics with Ben Grinstein, Donal O'Connell, Mark Wise and Terry Tomboulis. This work has been supported, in part, by the National Science Foundation under Grants No. NSF PHY1520292 and No. PHY1820675 (JFD), Conselho Nacional de Desenvolvimento Científico e Tecnológico-CNPq under Grant No. 307578/2015-1 (GM) and Fundação Carlos Chagas Filho de Amparo à Pesquisa do Estado do Rio de Janeiro-FAPERJ under Grant No. E-26/202.725/ 2018 (GM).
[1] T. D. Lee and G. C. Wick, Negative metric and the unitarity of the S matrix, Nucl. Phys. B9, 209 (1969).

[2] T. D. Lee and G. C. Wick, Unitarity in the $N \theta \theta$ sector of soluble model with indefinite metric, Nucl. Phys. B10, 1 (1969).

[3] T.D. Lee and G.C. Wick, Finite theory of quantum electrodynamics, Phys. Rev. D 2, 1033 (1970).

[4] R. E. Cutkosky, P. V. Landshoff, D. I. Olive, and J. C. Polkinghorne, A non-analytic S matrix, Nucl. Phys. B12, 281 (1969).

[5] D. G. Boulware and D. J. Gross, Lee-wick indefinite metric quantization: A functional integral approach, Nucl. Phys. B233, 1 (1984).

[6] S. Coleman, Acausality, in Erice 1969: Ettore Majorana Schoool on Subnuclear Phenomena, edited by A. Zicchici (Academic Press, New York, 1970), p. 282.

[7] B. Grinstein, D. O'Connell, and M. B. Wise, The Lee-Wick standard model, Phys. Rev. D 77, 025012 (2008).

[8] B. Grinstein, D. O'Connell, and M. B. Wise, Causality as an emergent macroscopic phenomenon: The Lee-Wick $\mathrm{O}(\mathrm{N})$ model, Phys. Rev. D 79, 105019 (2009).

[9] K. S. Stelle, Renormalization of higher derivative quantum gravity, Phys. Rev. D 16, 953 (1977).

[10] J. Julve and M. Tonin, Quantum gravity with higher derivative terms, Nuovo Cimento B 46, 137 (1978).

[11] E. S. Fradkin and A. A. Tseytlin, Renormalizable asymptotically free quantum theory of gravity, Phys. Lett. 104B, 377 (1981); B201, 469 (1982).

[12] E. Tomboulis, $1 / \mathrm{N}$ expansion and renormalization in quantum gravity, Phys. Lett. 70B, 361 (1977); E. T. Tomboulis, Renormalization and unitarity in higher derivative and nonlocal gravity theories, Mod. Phys. Lett. A 30, 1540005 (2015); I. Antoniadis and E. T. Tomboulis, Gauge invariance and unitarity in higher derivative quantum gravity, Phys. Rev. D 33, 2756 (1986).

[13] A. V. Smilga, Ghost-free higher-derivative theory, Phys. Lett. B 632, 433 (2006); A. Smilga, Classical and quantum dynamics of higher-derivative systems, Int. J. Mod. Phys. A 32, 1730025 (2017).

[14] M. B. Einhorn and D. R. T. Jones, Naturalness and dimensional transmutation in classically scale-invariant gravity,
J. High Energy Phys. 03 (2015) 047; D. R. T. Jones and M. Einhorn, Quantum gravity and dimensional transmutation, Proc. Sci., PLANCK2015 (2015) 061.

[15] A. Salvio and A. Strumia, Agravity, J. High Energy Phys. 06 (2014) 080; A. Salvio and A. Strumia, Agravity up to infinite energy, Eur. Phys. J. C 78, 124 (2018).

[16] J. F. Donoghue, Conformal model of gravitons, Phys. Rev. D 96, 044006 (2017); J. F. Donoghue, Quartic propagators, negative norms and the physical spectrum, Phys. Rev. D 96, 044007 (2017); J.F. Donoghue, Is the spin connection confined or condensed, Phys. Rev. D 96, 044003 (2017).

[17] J. F. Donoghue and G. Menezes, Gauge assisted quadratic gravity: A framework for UV complete quantum gravity, Phys. Rev. D 97, 126005 (2018); J. F. Donoghue and G. Menezes, Inducing the Einstein action in QCD-like theories, Phys. Rev. D 97, 056022 (2018).

[18] B. Holdom and J. Ren, QCD analogy for quantum gravity, Phys. Rev. D 93, 124030 (2016); B. Holdom and J. Ren, Quadratic gravity: From weak to strong, Int. J. Mod. Phys. D 25, 1643004 (2016).

[19] P. D. Mannheim, Unitarity of loop diagrams for the ghostlike $1 /\left(k^{2}-M_{1}^{2}\right)-1 /\left(k^{2}-M_{2}^{2}\right)$ propagator, Phys. Rev. D 98, 045014 (2018); P. D. Mannheim, Making the case for conformal gravity, Found. Phys. 42, 388 (2012).

[20] G. 't Hooft, The conformal constraint in canonical quantum gravity, arXiv:1011.0061; Local conformal symmetry: The missing symmetry component for space and time, Int. J. Mod. Phys. D 24, 1543001 (2015); Singularities, horizons, firewalls, and local conformal symmetry, Proc. Phys. 208, 12 (2018).

[21] S. D. Odintsov and I. L. Shapiro, General relativity as the low-energy limit in higher derivative quantum gravity, Classical Quantum Gravity 9, 873 (1992); Teor. Mat. Fiz. 90, 469 (1992) [Theor. Math. Phys. 90, 319 (1992)]; I. L. Shapiro and G. Cognola, Interaction of low-energy induced gravity with quantized matter and phase transition induced to curvature, Phys. Rev. D 51, 2775 (1995); I. L. Shapiro, Hilbert-Einstein action from induced gravity coupled with scalar field, Mod. Phys. Lett. A 09, 1985 (1994).

[22] G. Narain and R. Anishetty, Short distance freedom of quantum gravity, Phys. Lett. B 711, 128 (2012); G. Narain, 
Signs and Stability in Higher-Derivative Gravity, Int. J. Mod. Phys. A 33, 1850031 (2018).

[23] D. Anselmi, On the quantum field theory of the gravitational interactions, J. High Energy Phys. 06 (2017) 086; D. Anselmi, Fakeons and Lee-Wick Models, arXiv:1801 .00915; D. Anselmi and M. Piva, Perturbative unitarity of Lee-Wick quantum field theory, Phys. Rev. D 96, 045009 (2017).

[24] L. Alvarez-Gaume, A. Kehagias, C. Kounnas, D. Lüst, and A. Riotto, Aspects of quadratic gravity, Fortschr. Phys. 64, 176 (2016).

[25] L. Modesto and I. L. Shapiro, Superrenormalizable quantum gravity with complex ghosts, Phys. Lett. B 755, 279 (2016).

[26] A. Accioly, B. L. Giacchini, and I. L. Shapiro, Low-energy effects in a higher-derivative gravity model with real and complex massive poles, Phys. Rev. D 96, 104004 (2017).

[27] A. Salvio, Quadratic Gravity, Front. Phys. 6, 77 (2018).
[28] J. F. Donoghue, E. Golowich, and B. R. Holstein, Dynamics of the standard model (Cambridge University Press, Cambridge, England, 2014).

[29] G. Kallen, On the definition of the renormalization constants in quantum electrodynamics, Helv. Phys. Acta 25, 417 (1952).

[30] H. Lehmann, On the properties of propagation functions and renormalization contants of quantized fields, Nuovo Cimento 11, 342 (1954).

[31] E. Alvarez, L. Da Rold, C. Schat, and A. Szynkman, Vertex displacements for acausal particles: Testing the Lee-Wick Standard Model at the LHC, J. High Energy Phys. 10 (2009) 023.

[32] N. D. Birrell and P. C. W. Davies, Quantum fields in curved space (Cambridge University Press, Cambridge, England, 1982).

[33] I. L. Buchbinder, S. D. Odintsov, and I. L. Shapiro, Effective Action in Quantum Gravity (IOP, Bristol, UK, 1992). 